\title{
CUSTO DE PROCESSAMENTO DE PRODUTOS PARA SAÚDE: UMA REVISÃO INTEGRATIVA
}

\author{
Cost of healthcare products processing: an integrative review
}

\section{Costo de procesamiento de productos de salud: una revisión integrativa}

\author{
Rita Rozileide Nascimento Pereira ${ }^{1 *}$ (1D, Lívia Maia Pascoal ${ }^{2}$ (D), \\ Isaura Letícia Tavares Palmeiras Rolim ${ }^{3} \mathbb{D}$, Adriana Gomes Nogueira Ferreira² ${ }^{\mathbb{D}}$, Elza Lima da Silva ${ }^{3}$
}

RESUMO: Objetivo: Analisar o conhecimento produzido na última década sobre custos hospitalares relacionados ao processamento de produtos para saúde (PPS) reutilizáveis em Centros de Material e Esterilização (CME) e empresas processadoras. Método: Revisão integrativa da literatura, com buscas nas bases de dados Cumulative Index to Nursing and Allied Health Literature (CINAHL), Medical Literature Analysis and Retrieval System On-line (MEDLINE), Literatura Latino-Americana e do Caribe em Ciências da Saúde (LILACS), Scopus e Scientific Electronic Library Online (SciELO). Os artigos selecionados foram publicados entre 2009 e abril de 2019. A busca inicial resultou em 782 artigos e, após a aplicação dos critérios de inclusão e exclusão, a amostra final compôs-se de 15 estudos, que foram analisados na íntegra. Resultados: A maioria dos trabalhos comparou o custo de diferentes tecnologias empregadas no processamento de PPS para obter economia, ou avaliou a redução de gastos com desperdício, principalmente com a diminuição do tamanho de bandejas cirúrgicas para redução de custo com o processo. Conclusão: $O$ processamento de PPS mostrou ser medida que gera economia quando se selecionam adequadamente as tecnologias envolvidas no processo e se evitam os desperdícios. Palavras-chave: Custos hospitalares. Gestão em saúde. Economia da enfermagem. Esterilização.

ABSTRACT: Objective: To analyze the knowledge produced in the last decade on hospital costs related to the processing of reusable healthcare products (HP) performed in Sterile Processing Departments (SPD) and processing companies. Method: Integrative literature review, with searches in the following databases: Cumulative Index to Nursing and Allied Health Literature (CINAHL), Medical Literature Analysis and Retrieval System Online (MEDLINE), Latin American and Caribbean Health Sciences Literature (LILACS), Scopus, and Scientific Electronic Library Online (SciELO). The selected articles were published between 2009 and April 2019. The initial search resulted in 782 articles and, after applying the inclusion and exclusion criteria, the final sample consisted of 15 studies, which were analyzed in full. Results: Authors of most studies compared the cost of different technologies employed in the processing of HP to achieve savings, or evaluated the reduction of waste-related expenditures, mainly with the reduction of the size of surgery trays to reduce the processing cost. Conclusion: The processing of HP proved to be a measure that promotes economy when properly selecting the technologies involved in the process and avoiding waste.

Keywords: Hospital costs. Health management. Economics, nursing. Sterilization.

RESUMEN: Objetivo: Analizar el conocimiento producido, en la última década, sobre los costos hospitalarios relacionados con el procesamiento de productos de salud (PS) reutilizables en los Centros de Materiales y Esterilización (CME) y las empresas de procesamiento. Método: revisión integral de la literatura, búsqueda en las bases de datos CINAHL, MEDLINE, LILACS, SCOPUS y SciELO. Los artículos seleccionados se publicaron entre 2009 y abril de 2019. La búsqueda inicial resultó en 782 artículos y, después de aplicar los criterios de inclusión y exclusión, la muestra final consistió en 15 estudios, que se analizaron en su totalidad. Resultados: La mayoría de los estudios compararon el costo de diferentes tecnologías utilizadas en el procesamiento de PS para obtener ahorros o evaluaron la reducción de los gastos de residuos, principalmente con la reducción del tamaño de las bandejas quirúrgicas, para reducir el costo del proceso. Conclusión: El procesamiento de PS demostró ser una medida que genera ahorros, cuando las tecnologías involucradas en el proceso se seleccionan adecuadamente y se evitan los desperdicios. Palabras clave: Costos de hospital. Gestión en salud. Economía de la enfermería. Esterilización.

Mestranda em Enfermagem da Universidade Federal do Maranhão (UFMA) - São Luís (MA), Brasil.

2Professora do Departamento de Enfermagem da UFMA - Imperatriz (MA), Brasil.

${ }^{3}$ Professora do Departamento de Enfermagem da UFMA - São Luís (MA), Brasil.

*Autora correspondente: rrnpereira@hotmail.com

Recebido: 25/09/2019 - Aprovado: 24/03/2020

DOl: 10.5327/Z1414-4425202000020007 


\section{INTRODUÇÃO}

O Centro de Material e Esterilização (CME) é parte integrante do complexo hospitalar, inerente, indispensável e vital ao processo assistencial. É considerado uma unidade de apoio técnico, cuja finalidade é o fornecimento de produtos para saúde (PPS) adequadamente processados, proporcionando condições seguras para assistência à saúde dos indivíduos enfermos e sadios ${ }^{1}$.

Dada a crescente elevação dos custos na saúde, as instituições hospitalares enfrentam um desafio constante, o que torna necessária aos profissionais do $\mathrm{CME}$ a aquisição de conhecimentos sobre conceitos e técnicas da contabilidade de custos como ferramenta para gestão dos recursos ${ }^{2}$.

O gerenciamento de custo é um processo administrativo, que objetiva a tomada de decisão sobre a distribuição de recursos disponíveis de forma racionalizada e eficiente. Busca alcançar resultados que atendam às finalidades da instituição, por meio dos conhecimentos de análise econômica que oportunizem a escolha de decisões mais assertivas ${ }^{3}$.

O desperdício na área de Saúde agrava as dificuldades financeiras já existentes em razão da escassez de recursos, e caracteriza-se pelos gastos desnecessários, pelo uso de recursos disponíveis de forma descontrolada, irracional e inconsequente na produção de processos, produtos ou procedimentos destinados à assistência aos clientes ${ }^{4}$.

Dessa forma, os enfermeiros nesse momento devem ter conhecimento a respeito de custos hospitalares e dominar, detalhadamente, as atividades que compõem os processos de trabalho desenvolvidos, com a finalidade de aperfeiçoar aquelas que agregam valor ao produto final e eliminar as que são desnecessárias, sem prejuízo da qualidade do resultado do processamento ${ }^{5}$.

Ainda que reduzir custos em serviços de saúde seja tarefa difícil pela natureza do atendimento prestado, as decisões em Saúde, aliadas a princípios econômicos, ganham cada vez mais força, quando se considera a desproporção entre os recursos e as demandas crescentes da população ${ }^{6}$.

Assim, destaca-se a relevância do CME para a qualidade e os custos da assistência à saúde. Tornam-se necessárias a reflexão e a análise econômica acerca dos custos que incidem sobre os PPS processados em CME ou em empresa processadora, a fim de delinear o planejamento e a tomada de decisão pelo enfermeiro gestor.

\section{OBJETIVO}

Analisar o conhecimento produzido na última década sobre custos hospitalares relacionados ao processamento de produtos para saúde reutilizáveis em CME e empresas processadoras.

\section{MÉTODO}

Trata-se de estudo de revisão integrativa da literatura, desenvolvido com a finalidade de reunir e sintetizar trabalhos relativos ao tema investigado. Esse tipo de revisão define o conhecimento atual sobre uma temática específica, pois é conduzido de modo a identificar, analisar e sintetizar resultados de publicações independentes sobre o mesmo assunto 7 .

Para conferir criticidade científica a este estudo, seguiram-se as seguintes etapas: identificação do problema ou questão de pesquisa; busca na literatura, que incluiu a definição dos critérios de inclusão e exclusão dos artigos; avaliação dos dados para definição das informações a serem extraídas dos artigos selecionados; análise crítica dos estudos incluídos; e apresentação da revisão integrativa ${ }^{8}$.

Definiu-se como questão norteadora: qual é o conhecimento produzido, segundo a literatura, sobre custos hospitalares relacionados ao processamento de produtos para saúde reutilizáveis em CME e empresas processadoras?

O levantamento bibliográfico ocorreu durante o mês de abril de 2019 e foi realizado nas bases: Cumulative Index to Nursing and Allied Health Literature (CINAHL), com base na plataforma EBSCOhost; Medical Literature Analysis and Retrieval Sistem on-line (MEDLINE), com base na plataforma PubMed; Literatura Latino-Americana e do Caribe em Ciências da Saúde (LILACS); Scopus, da Elsevier; e Scientific Electronic Library Online (SciELO).

Os descritores para realizar a busca foram os termos contemplados nos Descritores em Ciências da Saúde (DeCS) e Medical Subject Headings (MeSH), quais sejam: administração de materiais no hospital/administración de materiales de hospital/materials management, hospital; esterilização/esterilización/sterilization; custos e análise de custo/costos y análisis de costo/costs and cost analysis; e economia da enfermagem/economía de la enfermería/economics, nursing.

Realizou-se o cruzamento dos descritores utilizando-se o operador booleano AND, nas seguintes combinações: materials management, hospital AND sterilization; materials management, hospital AND costs and cost analysis; sterilization AND costs and cost analysis; sterilization AND economics, nursing. Empregaram-se os filtros disponíveis em texto completo e nos idiomas português, inglês e espanhol.

Os artigos da amostra foram aqueles publicados nos últimos 10 anos, entre 2009 e abril de 2019, que estavam disponíveis 
gratuitamente e eram apresentados em texto completo e nos idiomas inglês, português e espanhol, cujo título e / ou resumo fizessem referência à temática. Excluíram-se revisões de literatura, cartas, editoriais, teses, dissertações e monografias.

Inicialmente analisou-se o título e/ou o resumo, como mostra a Figura 1.

Para a coleta de dados, utilizou-se instrumento adaptado do modelo validado por Ursi e Galvão 9 , que contemplou os seguintes itens: identificação do artigo original, características metodológicas do estudo, avaliação do rigor metodológico, intervenções estudadas e resultados encontrados.
Compilaram-se os dados extraídos dos trabalhos incluídos na pesquisa de forma descritiva em quadro previamente elaborado, que contemplou os seguintes aspectos: nome do artigo; autores e ano de publicação; objetivos; resultados; conclusões. Realizou-se síntese descritiva dos dados advindos da busca.

\section{RESULTADOS}

Dos 15 artigos analisados, sete (46\%) foram selecionados da base MEDLINE, quatro (27\%) da LILACS, três (20\%) da

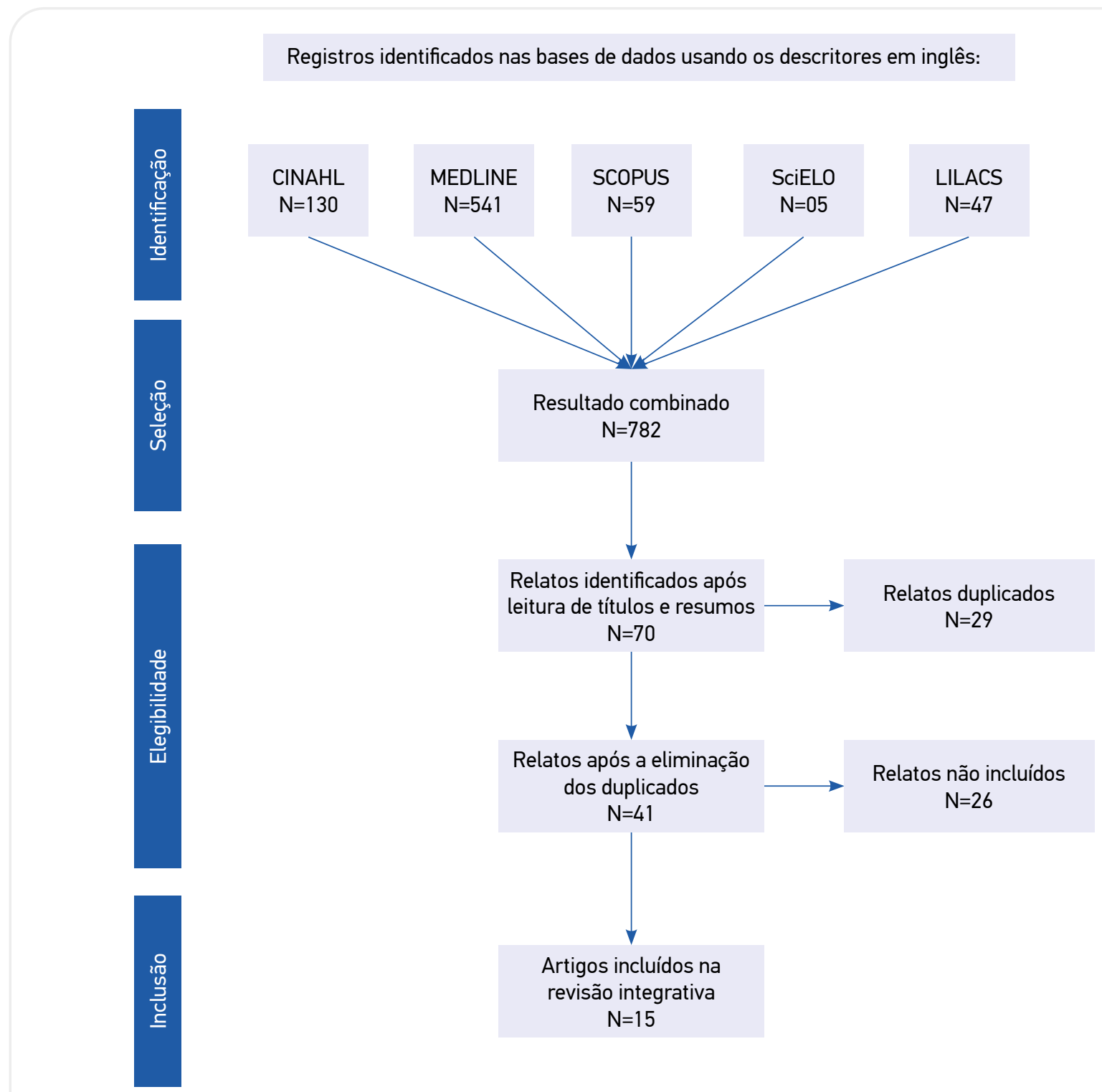

CINAHL: Cumulative Index to Nursing and Allied Health Literature; MEDLINE: Medical Literature Analysis and Retrieval System On-line; SciELO: Scientific Electronic Library Online; LILACS: Literatura Latino-Americana e do Caribe em Ciências da Saúde.

Figura 1. Processo de seleção de inclusão de estudos na revisão integrativa. 
CINAHL e um (7\%) da Scopus. Nenhum artigo se vinculou à base SciELO, pois os que foram encontrados nela encontravam-se repetidos em outras bases. Em relação aos locais das pesquisas, sete (46\%) foram desenvolvidas nos Estados Unidos, seis (40\%) no Brasil, uma (7\%) na Alemanha e uma (7\%) em Uganda. Nove (60\%) artigos foram publicados na língua inglesa e seis (40\%) na língua portuguesa.
Com base na análise realizada dos artigos selecionados, foi possível categorizar os estudos em três temáticas: avaliação dos custos de diferentes tecnologias usadas em processamento de PPS, avaliação da redução de desperdício no processamento de PPS e comparação do custo do uso de produtos reutilizáveis e de uso único. Cada uma das temáticas se encontra representada nos Quadros 1, 2 e 3.

Quadro 1. Avaliação dos custos de diferentes tecnologias usadas em processamento de produtos para saúde.

\begin{tabular}{|c|c|c|c|c|}
\hline $\begin{array}{l}\text { Autores, ano, } \\
\text { país }\end{array}$ & Objetivo & Método & Resultados & Conclusões \\
\hline $\begin{array}{l}\text { Souza et al., } \\
2015 \text {, Brasil }{ }^{1}\end{array}$ & $\begin{array}{l}\text { Avaliar a aplicação do } \\
\text { sistema de custeio ABC, } \\
\text { no CME de instituição } \\
\text { hospitalar pública. }\end{array}$ & $\begin{array}{l}\text { Pesquisa aplicada, } \\
\text { descritiva, } \\
\text { exploratória, do tipo } \\
\text { estudo de caso. }\end{array}$ & $\begin{array}{l}\text { Os valores dos objetos de custo } \\
\text { foram: desinfetado, } \mathrm{R} \$ 3,03 ; \\
\text { esterilizado, } \mathrm{R} \$ 6,05 \text {; pacote leve/ } \\
\text { avulso, } \mathrm{R} \$ 4,46 \text {; caixa/roupa } \\
\text { pequena, } \mathrm{R} \$ 6,34 \text { e caixa/roupa } \\
\text { média, } \mathrm{R} \$ 6,18 \text {. } 0 \text { objeto de custo } \\
\text { da caixa grande atingiu } \mathrm{R} \$ 14,16 \text {. }\end{array}$ & $\begin{array}{l}0 \text { custeio ABC é } \\
\text { ferramenta eficiente } \\
\text { para a prática } \\
\text { gerencial baseada em } \\
\text { evidências em CME. }\end{array}$ \\
\hline $\begin{array}{l}\text { Vital et al., } \\
2016 \text {, Brasil }\end{array}$ & $\begin{array}{l}\text { Analisar o custo das } \\
\text { embalagens utilizadas } \\
\text { no CME por meio } \\
\text { do método ABC. }\end{array}$ & $\begin{array}{l}\text { Estudo descritivo, } \\
\text { observacional, com } \\
\text { análise documental. }\end{array}$ & $\begin{array}{l}\text { Uma embalagem de tecido de algodão } \\
\text { custou, em um processamento, de } \\
\mathrm{R} \$ 9,309(40 \times 40 \mathrm{~cm}) \text { a } \mathrm{R} \$ 13,517 \\
\quad(1,4 \times 1,4 \mathrm{~m}) \text {. } \\
\text { Os valores encontrados para } \\
\text { embalagem dupla de grau cirúrgico } \\
\text { foram de } \mathrm{R} \$ 1,45(20 \times 40 \mathrm{~cm}) \text { a } \mathrm{R} \$ 1,32 \\
(20 \times 50 \mathrm{~cm}, 30 \times 30 \mathrm{~cm} \text { e } 30 \times 40 \mathrm{~cm}) .\end{array}$ & $\begin{array}{l}\text { A utilização de } \\
\text { embalagens de grau } \\
\text { cirúrgico torna-se } \\
\text { medida mais econômica } \\
\text { para a instituição. }\end{array}$ \\
\hline $\begin{array}{l}\text { Krohn } \\
\text { et al., } 2019 \text {, } \\
\text { Alemanha }^{10}\end{array}$ & $\begin{array}{l}\text { Avaliar os custos } \\
\text { de quatro sistemas } \\
\text { de embalagens, } \\
\text { considerando que a } \\
\text { qualidade da esterilidade } \\
\text { é idêntica para os } \\
\text { respectivos sistemas. }\end{array}$ & $\begin{array}{l}\text { Estudo analítico. } \\
\text { Aplicaram-se } \\
\text { testes estatísticos } \\
\text { e utilizou-se o } \\
\text { software EasyFit } \\
\text { Professional } \\
\text { version } 5.6 .\end{array}$ & $\begin{array}{l}\text { A embalagem contêiner sem } \\
\text { invólucro interno se mostrou a opção } \\
\text { mais econômica, a 2,05 euros. } \\
\text { A opção de duas folhas não tecido se } \\
\text { mostrou mais cara, a 3,87 euros. }\end{array}$ & $\begin{array}{l}\text { Diferentes alternativas } \\
\text { de embalagens para } \\
\text { esterilização fazem } \\
\text { diferença em tempo } \\
\text { e custos. Cada CME } \\
\text { deve analisar sua } \\
\text { própria situação. }\end{array}$ \\
\hline $\begin{array}{l}\text { Stipanich } \\
\text { et al., } 2018, \\
\text { Brasil }^{11}\end{array}$ & $\begin{array}{l}\text { Comparar os custos de } \\
\text { diferentes processos } \\
\text { de abastecimento } \\
\text { dos materiais de } \\
\text { terapia respiratória } \\
\text { em hospital geral. }\end{array}$ & $\begin{array}{l}\text { Estudo } \\
\text { observacional, com } \\
\text { revisão documental. }\end{array}$ & $\begin{array}{c}\text { A aquisição de materiais } \\
\text { permanentes com higienização no } \\
\text { CME-INST foi o processamento } \\
\text { dispendioso. A maior diferença } \\
\text { em valores deu-se no reanimador } \\
\text { manual, R\$ 1,10 (CME-INST), R\$ 1,98 } \\
\text { (CME-EXT) e R\$ 26,70 (MD); e a } \\
\text { menor diferença nos circuitos de } \\
\text { VMI R\$ 1,77 (CME-INST), R\$ 5,52 } \\
\text { (CME-EXT) e R\$ 7,04 (MD). }\end{array}$ & $\begin{array}{l}\text { O processo de } \\
\text { abastecimento } \\
\text { realizado no CME- } \\
\text { INST se mostrou mais } \\
\text { vantajoso, com menores } \\
\text { custos, em relação à } \\
\text { higienização em CME- } \\
\text { EXT e aquisição de MD. }\end{array}$ \\
\hline $\begin{array}{l}\text { McCreanor } \\
\text { e Graves, } \\
2017, \text { Estados } \\
\text { Unidos }^{12}\end{array}$ & $\begin{array}{c}\text { Avaliar economicamente } \\
\text { a esterilização } \\
\text { de materiais } \\
\text { termossensíveis, } \\
\text { principalmente } \\
\text { endoscópicos, usando } \\
\text { a baixa temperatura, } \\
\text { por plasma de peróxido } \\
\text { de hidrogênio, em vez } \\
\text { de autoclave a vapor. }\end{array}$ & $\begin{array}{l}\text { Estudo analítico que } \\
\text { usou a simulação } \\
\text { de Monte Carlos. }\end{array}$ & $\begin{array}{l}\text { A esterilização a baixa temperatura } \\
\text { é mais cara do que pelo vapor, no } \\
\text { entanto economias são realizadas, } \\
\text { em longo prazo, no reparo dos } \\
\text { instrumentos. Com base nos cálculos } \\
\text { do modelo, essas economias } \\
\text { provavelmente estarão na ordem } \\
\text { de US\$ 738,832 em } 10 \text { anos. }\end{array}$ & $\begin{array}{l}\text { Investimentos em } \\
\text { sistema de baixa } \\
\text { temperatura geram } \\
\text { economia em longo } \\
\text { prazo, pela redução da } \\
\text { necessidade de reparos } \\
\text { para instrumentos. }\end{array}$ \\
\hline
\end{tabular}


Quadro 1. Continuação.

\begin{tabular}{|c|c|c|c|c|}
\hline $\begin{array}{l}\text { Autores, ano, } \\
\text { país }\end{array}$ & Objetivo & Método & Resultados & Conclusões \\
\hline $\begin{array}{l}\text { Jerico e } \\
\text { Castilho, 2010, } \\
\text { Brasil }^{13}\end{array}$ & $\begin{array}{l}\text { Identificar o custo } \\
\text { dos processos } \\
\text { de desinfecção e } \\
\text { esterilização de } \\
\text { artigos hospitalares. }\end{array}$ & $\begin{array}{c}\text { Estudo exploratório, } \\
\text { descritivo, na } \\
\text { modalidade estudo } \\
\text { de caso. Adotou-se } \\
\text { o modelo ABM. }\end{array}$ & $\begin{array}{l}\text { Custos por ciclo/carga de } \\
\text { processamento: Desinfecção física, } \\
\text { US\$ 12,63; Desinfecção química, } \\
\text { US\$ 9,95; Esterilização VBTF, } \\
\text { US\$ 255,28; e Esterilização VSP, } \\
\text { US\$ 31,37. Custo por grupo de } \\
\text { produto: termossensível semicrítico, } \\
\text { US\$ 0,28, o menor valor, e } \\
\text { termorresistente crítico instrumental, } \\
\text { US\$ 1,75, o maior valor encontrado. }\end{array}$ & $\begin{array}{c}\text { A aplicação do ABM } \\
\text { no CME investigado } \\
\text { é exequível para o } \\
\text { gerenciamento de custos. }\end{array}$ \\
\hline
\end{tabular}

CME: Centro de Material e Esterilização; CME-INST: Centro de Material e Esterilização institucional; CME-EXT: Centro de Material e Esterilização terceirizado; MD: material descartável; VMI: Ventilação Mecânica Invasiva; ABM: activity-based management; ABC: custeio baseado em atividades; VBTF: Vapor a Baixa Temperatura de Formaldeído; VSP: Vapor Saturado sob Pressão.

Quadro 2. Avaliação da redução de desperdício no processamento de produtos para saúde.

\begin{tabular}{|c|c|c|c|c|}
\hline Autores, ano, país & Objetivo & Método & Resultados & Conclusões \\
\hline $\begin{array}{l}\text { Nast e Swords, } \\
2019, \text { Estados } \\
\text { Unidos }^{14}\end{array}$ & $\begin{array}{l}\text { Reduzir bandejas de } \\
\text { urologia de modo } \\
\text { que mais de } 50 \% \\
\text { dos instrumentos } \\
\text { serão usados, } \\
\text { levando a redução } \\
\text { de custos. }\end{array}$ & $\begin{array}{l}\text { Estudo prospectivo } \\
\text { analítico. Aplicou-se } \\
\text { o teste } t \text { de Student. }\end{array}$ & $\begin{array}{l}\text { Encontraram-se desde bandejas com } \\
\text { taxa de instrumentais usados de } 21,1 \% \\
\text { antes da redução, aumentada para } 48,2 \% \\
\text { após a redução; até bandejas com taxa } \\
\text { de utilização de } 41,9 \% \text { antes da redução, } \\
\text { com elevação da taxa de uso para } 71,7 \% \\
\text { após redução. Calculou-se economia de } \\
\text { US } \$ 7,48 \text { a US\$ } 70,18 \text { por procedimento. }\end{array}$ & $\begin{array}{l}\text { A iniciativa de } \\
\text { redução do } \\
\text { tamanho das } \\
\text { bandejas cirúrgicas } \\
\text { se mostrou } \\
\text { oportunidade de } \\
\text { redução de custos. }\end{array}$ \\
\hline $\begin{array}{l}\text { Cichos et al., 2017, } \\
\text { Estados Unidos }{ }^{15}\end{array}$ & $\begin{array}{l}\text { Mostrar o efeito que } \\
\text { a padronização de } \\
\text { bandejas cirúrgicas } \\
\text { tem sobre o número } \\
\text { de instrumentais } \\
\text { esterilizados e o } \\
\text { impacto sobre os } \\
\text { custos em hospital } \\
\text { universitário. }\end{array}$ & $\begin{array}{l}\text { Estudo de caso, } \\
\text { utilizando a } \\
\text { metodologia lean. }\end{array}$ & $\begin{array}{l}\text { Os resultados variaram de bandejas } \\
\text { que continham } 79 \text { instrumentais e } \\
\text { foram diminuídas para } 59 \text { ( } 75 \%) \text {, } \\
\text { até bandejas que continham } 113 \text { e } \\
\text { passaram a ter } 50 \text { instrumentais } \\
\text { (44\%). A economia estimada variou } \\
\text { de US\$55 por cada toracoscopia a } \\
\text { US\$ } 96 \text { por cada torocotomia. }\end{array}$ & $\begin{array}{l}\text { A redução do } \\
\text { processamento } \\
\text { de instrumentais } \\
\text { não usados reduz } \\
\text { custos e o peso das } \\
\text { bandejas, podendo } \\
\text { diminuir a incidência } \\
\text { de carga molhada. }\end{array}$ \\
\hline $\begin{array}{l}\text { Isaacson et al., } \\
\text { 2017, Estados } \\
\text { Unidos }^{16}\end{array}$ & $\begin{array}{l}\text { Caracterizar todos } \\
\text { os aspectos de } \\
\text { recursos utilizados } \\
\text { no processamento } \\
\text { de ureteroscópios } \\
\text { flexíveis } \\
\text { reutilizáveis, para } \\
\text { propor métodos de } \\
\text { redução de custo. }\end{array}$ & $\begin{array}{l}\text { Estudo prospectivo } \\
\text { e observacional. } \\
\text { Aplicou-se } \\
\text { o método de } \\
\text { custeio ABC. }\end{array}$ & $\begin{array}{l}0 \text { tempo total médio do processamento } \\
\text { individual foi } 229 \pm 74,4 \text { min, incluindo } \\
47,7 \text { min no serviço de endoscopia e } \\
126,5 \pm 55,7 \text { min de secagem. } \\
\text { O custo total de processamento de } \\
\text { um ureteroscópio foi US } \$ 96,13 \text {. }\end{array}$ & $\begin{array}{l}\text { Embora os custos } \\
\text { de reparos sejam o } \\
\text { principal caminho } \\
\text { para a redução de } \\
\text { custo, os autores } \\
\text { chamam a atenção } \\
\text { para a técnica de } \\
\text { secagem, que pode } \\
\text { reduzir o tempo } \\
\text { e os custos do } \\
\text { processamento. }\end{array}$ \\
\hline $\begin{array}{l}\text { Van Meter e Adam, } \\
\text { 2016, Estados } \\
\text { Unidos }^{17}\end{array}$ & $\begin{array}{l}\text { Identificar e estimar } \\
\text { os custos de } \\
\text { esterilização de } \\
\text { instrumentais não } \\
\text { usados em cirurgias } \\
\text { ginecológicas } \\
\text { eletivas. }\end{array}$ & $\begin{array}{l}\text { Estudo analítico, } \\
\text { observacional } \\
\text { e com coleta de } \\
\text { dados secundários. } \\
\text { Na análise } \\
\text { estatística usou-se } \\
\text { teste } z \text { para duas } \\
\text { proporções. }\end{array}$ & $\begin{array}{l}\text { O percentual de instrumentais usados } \\
\text { foi de } 20,5 \% \text {. } 0 \text { valor encontrado por } \\
\text { esterilização de instrumentais foi de } \\
\text { US\$ 3,19. Isso correlaciona-se com } \\
\text { US\$ 232,160 em desperdício com a } \\
\text { esterilização de instrumentais não usados. }\end{array}$ & $\begin{array}{l}\text { A diminuição de } \\
\text { instrumentais das } \\
\text { bandejas cirúrgicas, } \\
\text { em laparoscopia, } \\
\text { principalmente, tem } \\
\text { alto potencial para } \\
\text { redução de custos. }\end{array}$ \\
\hline
\end{tabular}


Quadro 2. Continuação.

\begin{tabular}{|c|c|c|c|c|}
\hline Autores, ano, país & Objetivo & Método & Resultados & Conclusões \\
\hline $\begin{array}{l}\text { Paula et al., 2015, } \\
\text { Brasil }^{18}\end{array}$ & $\begin{array}{c}\text { Quantificar } \\
\text { o número de } \\
\text { instrumentais } \\
\text { utilizados e não } \\
\text { utilizados durante } \\
\text { a cirurgia e estimar } \\
\text { os custos com } \\
\text { o processo de } \\
\text { esterilização. }\end{array}$ & $\begin{array}{l}\text { Estudo quantitativo, } \\
\text { descritivo, } \\
\text { de campo e } \\
\text { observacional. } \\
\text { Também utilizou } \\
\text { dados secundários. }\end{array}$ & $\begin{array}{l}\text { A média de desaproveitamento dos } \\
\text { instrumentais de foi de } 52 \% \text {. Houve } \\
\text { média de desperdício por caixa cirúrgica } \\
\text { de } \mathrm{R} \$ 7,28 \text {, no caso de parto cesáreo, } \\
\text { à média de } \mathrm{R} \$ 9,71 \text { por caixa usada } \\
\text { em cirurgias de histerectomia. }\end{array}$ & $\begin{array}{l}\text { Em um mês, ter- } \\
\text { se-ia, em média, } \\
\text { desperdício de } \\
\text { R\$ } 1.584,17 \text { com } \\
\text { a esterilização de } \\
\text { instrumentais que } \\
\text { não são utilizados } \\
\text { nas cirurgias. }\end{array}$ \\
\hline $\begin{array}{l}\text { Stockert e } \\
\text { Langerman, 2014, } \\
\text { Estados Unidos }^{19}\end{array}$ & $\begin{array}{l}\text { Demonstrar que } \\
\text { instrumentais não } \\
\text { usados custam um } \\
\text { valor não trivial no } \\
\text { CME da instituição. }\end{array}$ & $\begin{array}{l}\text { Estudo } \\
\text { observacional. } \\
\text { Realizou estatística } \\
\text { descritiva e } \\
\text { regressão linear } \\
\text { para análise } \\
\text { dos dados. }\end{array}$ & $\begin{array}{l}\text { A maior taxa de uso foi } 21,9 \% \text { em } \\
\text { neurocirurgias. } 0 \text { custo médio de } \\
\text { processamento de instrumentais } \\
\text { pode variar de US } 0,10 \text { a US } \$ 0,51 \\
\text { ou mais por instrumento. }\end{array}$ & $\begin{array}{l}\text { A atenção para a } \\
\text { composição das } \\
\text { bandejas cirúrgicas } \\
\text { deve resultar } \\
\text { em imediata } \\
\text { e significativa } \\
\text { economia no } \\
\text { trabalho do CME. }\end{array}$ \\
\hline
\end{tabular}

ABC: custeio baseado em atividades; CME: Centro de Material e Esterilização.

Quadro 3. Comparação dos custos do uso de produtos reutilizáveis e de uso único.

\begin{tabular}{|c|c|c|c|c|}
\hline Autor & Objetivo & Método & Resultados & Conclusões \\
\hline $\begin{array}{l}\text { Tomé e Lima, } \\
\text { 2015, } \text { Brasil }^{6}\end{array}$ & $\begin{array}{l}\text { Identificar o } \\
\text { custo direto do } \\
\text { reprocessamento de } \\
\text { campos de tecido de } \\
\text { algodão integrantes } \\
\text { do LAP cirúrgico. }\end{array}$ & $\begin{array}{l}\text { Estudo de caso } \\
\text { quantitativo, } \\
\text { exploratório- } \\
\text { descritivo. }\end{array}$ & $\begin{array}{l}0 \text { custo médio mensal do uso de LAP } \\
\text { cirúrgico totaliza US\$13.987,08, enquanto } \\
\text { o custo médio mensal referente ao } \\
\text { consumo de kits cirúrgicos e campos } \\
\text { de mesa auxiliar avulsos descartáveis } \\
\text { corresponderia a US\$29.127,15. }\end{array}$ & $\begin{array}{l}\text { O custo obtido pelo } \\
\text { processamento de } \\
\text { campos de tecido de } \\
\text { algodão integrantes } \\
\text { dos pacotes de LAP } \\
\text { cirúrgico foi de US\$ } 9,72 \text {. }\end{array}$ \\
\hline $\begin{array}{l}\text { Kuznik } \\
\text { et al., } 2012 \text {, } \\
\text { Uganda }^{20}\end{array}$ & $\begin{array}{l}\text { Comparar os custos nos } \\
\text { serviços de circuncisão } \\
\text { médica masculina } \\
\text { usando equipamentos } \\
\text { reutilizáveis versus } \\
\text { kits descartáveis. }\end{array}$ & $\begin{array}{l}\text { Estudo } \\
\text { exploratório. }\end{array}$ & $\begin{array}{l}0 \text { custo médio de kits para circuncisão } \\
\text { reutilizáveis é US\$ } 8,46 \text {. } 0 \text { custo de } \\
\text { um kit descartável varia de US\$ } 15,60 \\
\text { a US\$ } 20,80 \text {. Portanto, a economia } \\
\text { média por kit reutilizável alcança de } \\
\text { US\$ } 7,14 \text { a US\$ } 12,34 \text { ou de } 46 \text { a } 59 \% \text {. }\end{array}$ & $\begin{array}{l}0 \text { uso de kits reutilizáveis } \\
\text { em procedimentos de } \\
\text { circuncisão médica } \\
\text { masculina resultam em } \\
\text { economia de } 46 \text { a } 59 \% \text {. }\end{array}$ \\
\hline $\begin{array}{l}\text { Yung et al., } \\
2010 \text {, Estados } \\
\text { Unidos }^{21}\end{array}$ & $\begin{array}{l}\text { Avaliar o custo total } \\
\text { do uso de tesouras } \\
\text { ultrassônicas } \\
\text { reutilizáveis e comparar } \\
\text { com os custos } \\
\text { de equipamentos } \\
\text { descartáveis. }\end{array}$ & $\begin{array}{l}\text { Estudo prospectivo. } \\
\text { A estatística } \\
\text { descritiva foi } \\
\text { realizada com } \\
\text { SAS 9.1.3 para } \\
\text { Windows. }\end{array}$ & $\begin{array}{l}\text { O custo de aquisição por tesoura } \\
\text { descartável foi US\$ } 307 \text { e o custo total de } \\
\text { reprocessamento foi US\$ } 43,73 \text { por uso. } \\
\text { O reúso de tesouras ultrassônicas resultou } \\
\text { em economia de US\$ } 196,40 \text { por caso. }\end{array}$ & $\begin{array}{l}0 \text { uso de tesoura } \\
\text { reutilizável é mais } \\
\text { econômico, com } \\
\text { o aumento do } \\
\text { número de usos. }\end{array}$ \\
\hline
\end{tabular}

LAP: pacote de laparotomia.

\section{DISCUSSÃO}

No percurso da assistência hospitalar, com a crescente elevação de custos na área da Saúde, vêm-se procurando alternativas para gerar economias em procedimentos a ela associados, fortalecendo a necessidade de conhecimento relacionado aos princípios básicos de contabilidade de custos pelos profissionais atuantes em $\mathrm{CME}$, principalmente enfermeiros. Portanto, esses profissionais devem utilizar o conhecimento no assunto como ferramenta gerencial para fundamentar os argumentos e as negociações com administradores hospitalares, criando oportunidades para melhorar a eficiência do desempenho do serviço, racionalizando recursos e acompanhando a produtividade de área ${ }^{22}$.

Em relação às embalagens para esterilização utilizadas para o processamento de PPS, o tecido de algodão é uma das embalagens mais antigas e, atualmente, é amplamente utilizado para esterilização a vapor saturado sob pressão, apresentando como vantagens economia, propriedade de memória e resistência, que se 
aproximam de níveis ideais ${ }^{23}$. Entretanto, na literatura, a economia da embalagem de campo de algodão não se confirmou, quando comparada ao custo da embalagem descartável de grau cirúrgico².

Resultado semelhante foi identificado em estudo realizado na Bahia, no qual a embalagem de tecido de algodão apresentou o maior custo mensal e a de papel grau cirúrgico o menor custo, quando comparados às outras embalagens. Isso desmistifica o paradigma que existe nos $\mathrm{CME}$ brasileiros de que o tecido de algodão seria a embalagem mais barata ${ }^{24}$.

O contêiner rígido é outra embalagem permanente que, ao mesmo tempo, acondiciona e protege os instrumentais, mantendo-os estéreis até o momento do seu uso; pode ser de alumínio, aço inoxidável ou plástico e utilizar filtros descartáveis ou reutilizáveis ${ }^{25}$. Uma desvantagem seria o custo elevado, contudo esse valor pode ser diluído pelo número de reutilizações, podendo gerar economia, conforme demonstrado em estudo ${ }^{10}$.

No uso do contêiner rígido, não se recomenda a utilização de outro tipo de embalagem dentro ou fora dele, pois isso pode dificultar a saída de ar, a penetração do agente esterilizante e a etapa de secagem ${ }^{25}$.

Evidências demonstram diferenças significativas de custos entre diferentes métodos de processamento de PPS usados em um CME, sendo a desinfecção de alto nível um processo mais barato que a esterilização, e a esterilização a vapor mais barata que a esterilização a baixa temperatura ${ }^{1,12,13}$.

Apesar de mais rentável, o uso excessivo e a manutenção deficiente dos equipamentos tornam a esterilização a vapor ineficiente ao longo do tempo. Todavia, a substituição desse método pela esterilização por plasma de peróxido de hidrogênio aumentaria os gastos associados aos procedimentos de esterilização para a unidade ${ }^{26}$. Assim, o uso simultâneo e adequado desses dois métodos, esterilização a vapor e peróxido de hidrogênio, pode ser mais conveniente ${ }^{26}$.

Sobre a gestão do CME, a terceirização do processamento de PPS, realizada pelas empresas processadoras, demonstrou ser opção mais cara que o processamento em CME institucional em estudo realizado com materiais de assistência ventilatória ${ }^{11}$.

A segurança da esterilização em CME institucional e em empresa terceirizada é a mesma, porém a esterilização externa tem maior custo do que outras opções e maior possibilidade de atraso no fornecimento ${ }^{27}$. Por outro lado, com a terceirização do processamento, há menor necessidade de pessoal treinado e suporte de insumos no hospital ${ }^{27}$.

A revisão de bandejas cirúrgicas, diminuindo-se o número de instrumentais que comumente são processados sem que sejam usados nos procedimentos cirúrgicos, tem sido uma das estratégias mais adotadas por serviços de cirurgia para diminuir desperdícios e, consequentemente, reduzir custos, gerando economias significativas. Esse fato foi apontado em estudos $^{14,15,17-19}$, sobretudo nos casos em que as bandejas de instrumentais eram utilizadas em cirurgias endoscópicas ${ }^{15,17}$, pelo motivo de os instrumentais requererem processamento mais elaborado e demorado ${ }^{17}$.

O desperdício está atrelado ao desenvolvimento de atividades que não favorecem o produto ou os serviços produzidos, apenas custos e despesas desnecessários ${ }^{4}$. Assim, a apuração das fontes de desperdícios relacionados aos recursos materiais, processos e pessoal constitui absoluta necessidade em organizações públicas e privadas. O excesso de etapas nos processos também pode gerar desperdício, causando ineficiência e atrasos no processo de trabalho ${ }^{4}$.

Além disso, a Association for the Advancement of Medical Instrumentation (AAMI) e a Association of periOperative Registered Nurses (AORN) associam o peso das bandejas de instrumentais a um risco maior de pacote molhado após a esterilização, recomendando peso máximo de 25 libras $^{28}$.

O uso de métodos de custeio, com base no mapeamento das etapas que compõem o processamento de materiais, tem sido utilizado como medida para reduzir custos em processamento de PPS, permitindo incorporar sugestões de redirecionamento de recursos ${ }^{16}$.

Os processos envolvidos nas organizações de saúde requerem avaliação e controle quanto à sua eficiência, produtividade e qualidade, pois questões relacionadas aos custos têm implicações para a quantidade de serviços prestados aos clientes e, por meio do mapeamento, torna-se possível a visualização dos recursos consumidos e, consequentemente, sua otimização ${ }^{5}$.

Apesar dos avanços tecnológicos na fabricação de PPS de uso único, ao se comparar economicamente o uso desses materiais aos produtos reutilizáveis equivalentes, estes últimos ainda são mais custo-efetivos, apesar do custo de processamento no $\mathrm{CME}^{11,20,21}$.

Vários dispositivos, como campos de algodão e instrumentais, são fabricados para permitir a reutilização até o limite de sua eficácia e funcionalidade, podendo levar à redução de custos e à redução na quantidade de resíduos gerados por itens de uso único. No entanto é necessário assegurar, no momento da escolha, que esses produtos permaneçam seguros para a utilização no paciente ${ }^{29}$.

Este estudo apresenta limitações por não poder avaliar os impactos ambientais causados por determinadas tecnologias usadas no processamento de PPS ou por produtos de uso único, bem como o impacto relacionado à segurança do paciente e à Infecção Relacionada à Assistência à Saúde (IRAS). 


\section{CONSIDERAÇÕES FINAIS}

A análise dos 15 artigos publicados em periódicos nacionais e internacionais demonstrou que o processamento de PPS em CME institucionais mostrou ser medida que gera economia quando há gestão de custos, ao se selecionarem adequadamente as tecnologias envolvidas no processo e se evitarem os desperdícios. Os resultados mostram que, apesar dos avanços tecnológicos na fabricação de materiais de uso único que substituam PPS reutilizáveis processados nos CME institucionais, o uso de materiais reutilizáveis ainda é a alternativa mais viável economicamente.
Ressaltamos que, embora a variável custo seja fator relevante, outros aspectos não financeiros devem ser considerados, como a segurança do paciente e os impactos ambientais que envolvem os diferentes métodos de processamento de PPS.

\section{AGRADECIMENTOS}

Agradecemos à Coordenação de Aperfeiçoamento de Pessoal de Nível Superior (CAPES), código de financiamento 001.

\section{REFERÊNCIAS}

1. Souza WR, Spiri WC, Lima SAM, Bernardes A, Luppi CHB. Utilização do custeio baseado em atividades em centro de material e esterilização como ferramenta gerencial. Rev Eletr Enf [Internet]. 2015 [acessado em 14 abr. 2019];17(2):290-301. Disponível em: http://dx.doi.org/10.5216/ree.v17i2.27540

2. Vital JS, Miranda LN, Nagliate PC, Vasconcelos EL. Analysis of cost of sterilization packaging using costing based activities. J Nurs UFPE On Line [Internet]. 2016 [acessado em 14 abr. 2019];10(8):2877-85. Disponível em: http://doi.org/10.5205/ reuol.9373-82134-1-RV1008201613

3. Oliveira DF, Dedavid BA. Transformações ocorridas no tecido de algodão utilizado como embalagem no processo de esterilização de materiais: um estudo de caso [dissertação]. Porto Alegre: Pontifícia Universidade Católica do Rio Grande do Sul; 2016. $105 \mathrm{p}$.

4. Castilho V, Castro LC, Couto AT, Maia FOM, Sasaki NY, Nomura $\mathrm{FH}$, et al. Survey of the major sources of waste in the health care units of a teaching hospital. Rev Esc Enfermagem USP. 2011;45(Núm. Esp.):1613-20. https://doi.org/10.1590/ S0080-62342011000700012

5. Tomé MF, Lima AFC. Mapeamento do processo de reprocessamento de campos cirúrgicos de tecido de algodão. Rev SOBECC. 2015;20(4):197-201. http://dx.doi.org/10.5327/ Z1414-4425201500040003

6. Tomé MF, Lima AFC. Custo direto de reprocessamento de campos cirúrgicos de tecido de algodão: um estudo de custo. Rev Esc Enferm USP. 2015;49(3):494-501. http://dx.doi.org/10.1590/ S0080-623420150000300018

7. Souza MTS, Silva MD, Carvalho R. Revisão Integrativa: o que é e como fazer. Einstein. 2010;8(n. 1):102-6. http://dx.doi.org/10.1590/ s1679-45082010rw1134

8. Whittemore R, Knafl K. The integrative review: updated methodology. J Adv Nurs. 2005;52(5):546-53. http://doi. org/10.1111/j.1365-2648.2005.03621.x
9. Ursi ES, Gavão CM. Prevenção de lesões de pele no perioperatório: revisão integrativa da literatura. Rev Latino-Am Enferm. 2006;14(1):124-31.

10. Krohn M, Fengler J, Mickley T, Flessa S. Analysis of processes and costs of alternative packaging options of sterile goods in hospitals: a case study in two German hospitals. Health Econ Rev. 2019;9(1):1. https://doi.org/10.1186/s13561-018-0218-2

11. Stipanich C, Goulardins JB, Medeiros M, Maria F, Tanaka C. Comparação de custos em diferentes processos de abastecimento de materiais de fisioterapia respiratória em um hospital público de porte extra. Rev Pesq Fisio. 2018;8(2):230-8. http://dx.doi.org/10.17267/2238-2704rpf.v8i2.1927

12. McCreanor V, Graves N. An economic analysis of the benefits of sterilizing medical instruments in low-temperature systems instead of steam. Am J Infect Control. 2017;45(7):756-60. https:// doi.org/10.1016/j.ajic.2017.02.026

13. Jerico MC, Castilho V. Gerenciamento de custos: aplicação do método de custeio baseado em atividades em centro de material esterilizado. Rev Esc Enferm USP. 2010;44(3):745-52. https://doi.org/10.1590/ S0080-62342010000300028

14. Nast K, Swords KA. Decreasing operating room costs via reduction of surgical instruments. J Pediatr Urol. 2019;15(2):153.e1-e6. http:// doi.org/10.1016/j.jpurol.2019.01.013

15. Cichos KH, Linsky PL, Wei B, Minnich DJ, Cerfolio RJ. Cost savings of standardization of thoracic surgical instruments: the process of Lean. Ann Thorac Surg. 2017;104(6):1889-95. http://dx.doi.org/10.1016/j. athoracsur.2017.06.064

16. Isaacson D, Ahmad BA, Metzler I, Tzou DT, Taguchi K, Usawachintachit $M$, et al. Defining the costs of reusable flexible ureteroscope reprocessing using time-driven activity-based costing. J Endourol. 2017;31(10):1026-31. http://doi.org/10.1089/end.2017.0463

17. Van Meter MM, Adam RA. Costs associated with instrument sterilization in gynecologic surgery. Am J Obstet Gynecol. 2016;215(5):652.e1-5. http://dx.doi.org/10.1016/j.ajog.2016.06.019 
18. Paula JRA, Silva RCR, Vedovato CA, Boaventura AP. Instrumentais nas caixas cirúrgicas: avaliação de custo. Rev SOBECC. 2015;20(2):7380. https://doi.org/10.5327/Z1414-4425201500020003

19. Stockert EW, Langerman A. Assessing the magnitude and costs of intraoperative inefficiencies attributable to surgical instrument trays. J Am Coll Surg. 2014;219(4):646-55. http://dx.doi.org/10.1016/j. jamcollsurg.2014.06.019

20. Kuznik A, Lamorde M, Sekavuga DB, Picho B, Coutinho A. Medical male circumcision for HIV/AIDS pevention in Uganda: the cost of disposable versus re-usable circumcision kits. Trop Doct. 2012;42(1):57. http://doi.org/10.1258/td.2011.110297

21. Yung E, Gagner M, Pomp A, Dakin G, Milone L, Strain G. Cost comparison of reusable and single-use ultrasonic shears for laparoscopic bariatric surgery. Obes Surg. 2010;20:512-8. http://doi.org/10.1007/ s11695-008-9723-4

22. Tome MF, Lima AFC. Custo do reprocessamento de campos cirúrgicos de tecido de algodão: um estudo de caso [dissertação]. São Paulo: Escola de Enfermagem da Universidade de São Paulo; 2014. 120 p.

23. Freitas LR, Tipple AFV, Felipe DP, Rodrigues NSR, Melo DS. Embalagem de tecido de algodão: análise do uso em hospitais de médio e grande porte. Rev Eletr Enfer [Internet]. 2012 [acessado em 28 mar. 2019];14(4):811-20. Disponível em: https://doi.org/10.5216/ree. v14i4.16612
24. Souza AS, Sória DAC, Araújo JO, Silva MT, Andrade NC. Embalagens para esterilização: suas aplicações e recomendações na prática hospitalar. Rev Pesq Cuid Fundam Online. 2010;2(Supl.):316-9. http:// dx.doi.org/10.9789/2175-5361.rpcfo.v0.931

25. Associação Brasileira de Enfermeiros de Centro Cirúrgico, Recuperação Anestésica e Centro de Material e Esterilização. Diretrizes de práticas em enfermagem cirúrgica e processamento de produtos para saúde. 7a ed. São Paulo: SOBECC; Barueri: Manole; 2017.

26. Sanjuana GOM, Iris CH, Federico MQJ. Análisis de costos em três métodos de esterilización. Rev Enferm IMMS [Internet]. 2006 [acessado em 28 jun. 2019];14(3):131-5. Disponivel em: https://www.medigraphic. com/pdfs/enfermeriaimss/eim-2006/eim063c.pdf

27. Dehnavieh R, Mirshekari N, Ghasemi S, Goudarzi R, Haghsoost AA, Mehrolhassani MH, et al. Health technology assessment: off-site sterilization. Medical J Islam Repub Iran. 2016;30:345.

28. Seavey R. High-level disinfection, sterilization, and antisepsis: current issues in reprocessing medical and surgical instruments. Am J Infect Control. 2013;41(5 Supl.):S111-7. http://dx.doi.org/10.1016/j. ajic.2012.09.030

29. Evangelista SS, Santos SG, Stoianoff MAR, Oliveira AC. Analysis of microbial load on surgical instruments after clinical use and following manual and automated cleaning. Am J Infect Control. 2015;43(5):5227. http://dx.doi.org/10.1016/j.ajic.2014.12.018 